\title{
An inverse Fast Multipole Method for imaging applications
}

\author{
Yuri Álvarez, Member, IEEE, José Ángel Martínez, Member, IEEE, Fernando Las-Heras, Senior Member, \\ IEEE, Carey M. Rappaport, Fellow, IEEE
}

\begin{abstract}
An inverse Fast Multipole Method (FMM) for imaging applications is presented. The goal is the acceleration of an inverse source-based method for geometry reconstruction achieved by taking advantage of the multipole expansion properties of the scattered fields and reconstructed equivalent currents. By conjugating the FMM terms, it is possible to recover the equivalent currents from the scattered fields in just one step rather than using matrix inversion or cost function minimization. For the sake of simplicity, 2D problems where the objects-undertest having symmetry along one dimension are considered.
\end{abstract}

Index Terms - Imaging, Inverse Methods, Fast Multipole Method, Near Field characterization, Sources Reconstruction Method (SRM).

\section{INTRODUCTION}

$\mathrm{I}_{\mathrm{o}}^{\mathrm{N}}$ NVERSE scattering methods are the basis for a wide range of applications in which non-destructive testing is required. Among these applications are medical diagnostics [1], detection of buried targets [2],[3] or security scanners for detecting concealed weapons [4].

The inverse scattering problem can be divided according to the targeted solution: either by profile or geometry retrieval (object-under-test external boundary) or by constitutive parameter retrieval.

In regard to the profile reconstruction, one of the possibilities is the parameterization of the object contour, thus imposing the proper boundary condition (e.g. zero tangential field in case of PEC) [5]-[7]. Further improvements could be

Manuscript received August 20, 2011. This work is supported by CenSSIS, the Gordon Center for Subsurface Sensing and Imaging Systems NSF ERC Program (Award number EEC-9986821); by the "Ministerio de Ciencia e Innovación” of Spain/FEDER under projects TEC2008-01638/TEC (INVEMTA), CONSOLIDER-INGENIO CSD2008-00068 (TERASENSE), and TEC2011-24492/TEC (iSCAT); by PCTI Asturias under projects EQUIP08-06, FC09-COF09-12, EQUIP10-31, and PC10-06 (FLEXANT); and by "Cátedra Telefónica - Universidad de Oviedo". This material is based upon work supported by the Science and Tech. Directorate, U.S. Department of Homeland Security under the Award Number "2008-ST-061-ED0001". The views and conclusions contained in this document are those of the authors and should not be interpreted as necessarily representing the official policies, either expressed on implied, of the U.S. Department of Homeland Security.

Y. Alvarez, and F. Las-Heras are with the Area of Signal Theory and Communications, Department of Electrical Engineering, Universidad de Oviedo, E-33203, Gijón, SPAIN. Phone: +34-985-182-541; fax: +34-985182-466 (e-mail: yalopez@tsc.uniovi.es ; flasheras@tsc.uniovi.es).

J. A. Martínez and C. M. Rappaport are with The Gordon CenSSIS, Northeastern University, Boston, MA 02115 USA (e-mail: jmartine@ece.neu.edu; rappapor@ece.neu.edu). made with the introduction of auxiliary equivalent currents inside the object contour, which would improve the estimation of the scattered field [8]. In both cases, the resulting system of equations is non-linear, requiring non-linear optimization methods for solution.

Other methods are based on the so-called inverse source problem [9]-[12], where a set of equivalent currents are reconstructed in order to retrieve geometry and/or constitutive parameters. This inverse problem was initially reduced to a linear system of equations relating the scattered field and the equivalent currents [9]. The improvement of the inverse scattering method accuracy results in a non-linear formulation of the inverse scattering problem. For a general case, the nonlinear problem must be solved using global search algorithms, as proposed in [10] using genetic algorithms, or with the Inexact-Newton method [11] .

Even when the proposed problem is linear, one of the obstacles to be overcome is the computational cost, especially in those cases where the observation and reconstruction domains are electrically large, as in the case of burgeoning applications of millimeter wave radars. Moreover, some of the practical applications require quasi real-time processing. To reduce the computational time, two strategies can be adopted: i) Hardware improvement, as for example, the use of Graphics Processing Units (GPU), and ii) Introduction of acceleration methods in the inversion algorithms.

This work is focused on the second strategy and consequently presents an inverse Fast Multipole Method (FMM) [13]-[17], which is applied to accelerate an existing Volumetric Source Reconstruction Method [12]. While the FMM has been successfully applied in radar imaging [18] and in the Source Reconstruction Method (SRM) [19],[20], this work also presents a new forward solution of the inverse problem (i.e. one not requiring any matrix inversion).

\section{The InVERSE FAst Multipole Method}

The Volumetric Source Reconstruction Method [12] is based on the volumetric equivalence principle that allows the substitution of the original sources (e.g. the induced currents on a metallic scatterer) with a volumetric equivalent current distribution that radiates the same field as the original sources.

For the sake of simplicity, the formulation will be given for a 2D case assuming a TM-polarized field as in [12]. The field radiated by an electric current distribution $\vec{J}_{e q}\left(\vec{\rho}^{\prime}\right)=J_{e q}\left(\vec{\rho}^{\prime}\right) \hat{z}$ defined on a surface $S$ ' is given by (1): 


$$
\vec{E}_{\text {scatt }}(\vec{\rho})=-\hat{z} \frac{j k_{0} \eta}{4} \int_{S^{\prime}}\left\{H_{0}^{(2)}\left(k_{0} R\left(\vec{\rho} ; \vec{\rho}^{\prime}\right)\right) J_{e q}\left(\vec{\rho}^{\prime}\right)\right\} d S^{\prime}
$$

Where $H_{0}^{(2)}$ is the Hankel function of 0 -th order and second kind, $R\left(\vec{\rho} ; \vec{\rho}^{\prime}\right)$ is the distance from the observation points $\vec{\rho}=\vec{\rho}(\rho, \phi)$ to the source points $\vec{\rho}^{\prime}=\vec{\rho}^{\prime}\left(\rho^{\prime}, \phi^{\prime}\right), k_{0}$ is the wavenumber, and $\eta$ is the intrinsic impedance. The integral equation above can be expressed as a linear system of equations where the impedance matrix $\left(\mathrm{Z}_{\text {Esatat, } \mathrm{eq}_{\mathrm{eq}}}\right)$ is approximated by the Fast Multipole Method (FMM) factors: disaggregation $(D)$, translation $(T)$, and aggregation $(A)[13]$ :

$$
\begin{aligned}
& \left(E_{\text {scatt }}\right)=\left(Z_{E_{\text {sat }}, J_{e q}}\right)\left(J_{e q}\right) \approx(D)(T)(A)\left(\hat{J}_{e q}\right) \\
& \hat{J}_{e q} \text { is defined as } \hat{J}_{e q}=J_{e q} \cdot \Delta S \text { where } \Delta S \text { is the subdomain }
\end{aligned}
$$
where the current is defined and $J_{e q}$ is the current density in that subdomain. As indicated in [19],[20] one of the FMM advantages when it is applied to inverse problems is the fact that the observation and source domains are physically different, so in most cases, no adjacent groups are present. Even more, if the distance between the observation and source domains is large enough, the FMM far-field condition [15], [19] is satisfied for all the groups, allowing for the translation matrix simplification.

The aggregation $(A)$ and disaggregation $(D)$ factors are based on plane wave expansions with respect to the center of each group. In the case of the translation matrix (T), the farfield approach [15] is considered for a 2D formulation (3),

$$
\begin{aligned}
& (A)=e^{-j k_{0} \hat{k} \cdot\left(\vec{C}_{s}-\vec{\rho}^{\prime}\right)} \quad(D)=e^{-j k_{0} \hat{k} \cdot\left(\vec{\rho}-\vec{C}_{o b s}\right)} \\
& (T)=e^{-j k_{0}\left|\vec{C}_{o b s}-\vec{C}_{s}\right|}\left(\left|\vec{C}_{o b s}-\vec{C}_{s}\right|\right)^{-1 / 2}
\end{aligned}
$$

where $\left|\vec{C}_{o b s}-\vec{C}_{s}\right|$ is the distance from the center of the source group $\vec{C}_{s}$ to the center of the observation group $\vec{C}_{\text {obs }}$.

The FMM can also be applied to the inverse scattering problem. As the $(A)$ and $(D)$ terms are plane wave operators, it is possible to reverse the propagation direction by conjugating these terms, resulting in $\left(D_{\mathrm{i}}\right)$ and $\left(A_{\mathrm{i}}\right)$. The direction of the translator operator can be reversed by doing the same while the attenuation factor is inverted, $\left(T_{\mathrm{i}}\right.$ ) (Eq. (4)). Thus, the electric currents can be calculated from the scattered fields as indicated in Eq. (5):

$$
\begin{aligned}
& \left(D_{i}\right)=e^{+j k_{0} \hat{k} \cdot\left(\vec{C}_{s}-\vec{\rho}^{\prime}\right)} \quad\left(A_{i}\right)=e^{+j k_{0} \hat{k} \cdot\left(\vec{\rho}-\vec{C}_{o b s}\right)} \\
& \left(T_{i}\right)=e^{+j k_{0}\left|\vec{C}_{o b s}-\vec{C}_{s}\right|}\left(\left|\vec{C}_{o b s}-\vec{C}_{s}\right|\right) \\
& \left(\hat{J}_{e q}\right) \approx\left(D_{i}\right)\left(T_{i}\right)\left(A_{i}\right)\left(E_{\text {scatt }}\right)
\end{aligned}
$$

Consequently, it is possible to recover the currents from the scattered fields in just one step without the use of matrix inversion or cost function minimization. The remarkable contribution of this approach is the computational cost savings. No linear system of equations needs to be solved with the currents calculated in a "forward" manner. In addition, because the FMM is an acceleration technique, it also contributes the reduction of the computational cost: while the impedance matrix $\left(\mathrm{Z}_{\text {Esat, Jeq }}\right)$ size is equal to the number of observation points times the number of sources, the inverse FMM is implemented in such a way so that just the translation matrix $\left(T_{\mathrm{i}}\right)$, whose size is the number of observation groups times the number of source groups, is stored.

The inverse FMM is based on the far-field approach of the translation term $\left(T_{\mathrm{i}}\right)$. As stated in [15], the applicability of this approach is based on the following criterion (6):

$$
\min \left\{\left|\vec{C}_{\text {obs }}-\vec{C}_{s}\right|\right\}>>\left(k_{0} \max \left\{D_{g}\right\}^{2}\right) / 2
$$

where $D_{\mathrm{g}}$ is the size of the observation and sources groups. Note that for electrically large objects, the observation domain may be placed in the near field region of the object, but by making adjustments based on the group size, the FMM farfield condition can be still satisfied.

When considering multiple frequencies $\left(N_{\text {freq }}\right)$ and incident plane waves $\left(M_{\mathrm{inc}}\right)$ illuminating the object-under-test [12], an inverse problem is solved independently for each incidence and frequency. The equivalent currents retrieved for each case are combined coherently [2] according to:

$$
\vec{J}\left(\vec{\rho}^{\prime}\right)=\sum_{m=1}^{M_{\text {inc }}} \sum_{n=1}^{N_{\text {freq }}} \vec{J}^{m, n}\left(\vec{\rho}^{\prime}\right) e^{+j k_{0}^{n}\left(\vec{\rho}^{\prime} \cdot \hat{k}_{\text {inc }}^{m}\right)}
$$

where $k_{0}^{n}$ is the wavenumber for the $n$-th frequency, and $\hat{k}_{i n c}^{m}$ is the unitary propagation vector for the $m$-th incident direction of the incident plane wave.

\section{APPLICATION EXAMPLES}

\section{A. Metallic object profile reconstruction}

In order to test the inverse FMM capabilities, an arbitrary object with a constant cross section on z-axis is chosen. The object is illuminated by $3 \mathrm{TM}$-polarized incident plane waves coming from $\phi=0^{\circ}, 120^{\circ}$ and $240^{\circ}$, with frequencies from 700 to $1000 \mathrm{MHz}$ in $20 \mathrm{MHz}$-step (35\% bandwidth with respect to the $850 \mathrm{MHz}$ center frequency). The scattered field for each incidence and frequency is simulated using 2D-Method-ofMoments software, where the field is evaluated on a circle with radius $\rho=10 \mathrm{~m}$, and sampled each $\Delta \phi=2^{\circ}$. The field on the observation points will be affected by numerical noise to simulate virtual measurements.

The reconstruction domain is a $5 \times 5.5 \mathrm{~m}$ rectangular domain discretized in $4 \times 4 \mathrm{~cm}$ square patches $(0.13 \times 0.13 \lambda$ at the highest frequency, $1 \mathrm{GHz}$ ). This means that the resulting system of equations has 360 equations (number of field samples acquired in the circumferential observation domain) and 17388 unknowns (number of sources domain patches). As the number of incidences and frequencies is $M_{\mathrm{inc}}=3$ and $N_{\text {freq }}=16$ respectively, 48 inverse problems have to be solved.

Regarding inverse FMM, no grouping is made on the observation domain (as only 360 points are considered), so the observation domain groups' size is zero. The sources domain is divided into 156 groups, each with a size of approximately $D_{\mathrm{g}}=0.6 \mathrm{~m}$. As the minimum distance from observation to source groups is $6.2 \mathrm{~m}$, then: 


$$
\min \left\{\left|\vec{C}_{\text {obs }}-\vec{C}_{s}\right|\right\} \approx 6.2 m \quad\left(k_{0} \max \left\{D_{g}\right\}^{2}\right) / 2 \approx 4 m
$$

So the FMM far-field criterion is (weakly) satisfied at the highest working frequency, $1 \mathrm{GHz}$. Fig. 1 represents the observation and source domains, and the inverse FMM grouping. With regard to the computational cost, Table 1 compares the calculation time of the Volumetric Source Reconstruction Method [12] and the Inverse FMM technique. Even with a 2D geometry, remarkable speed-up, about 40x, can be appreciated.

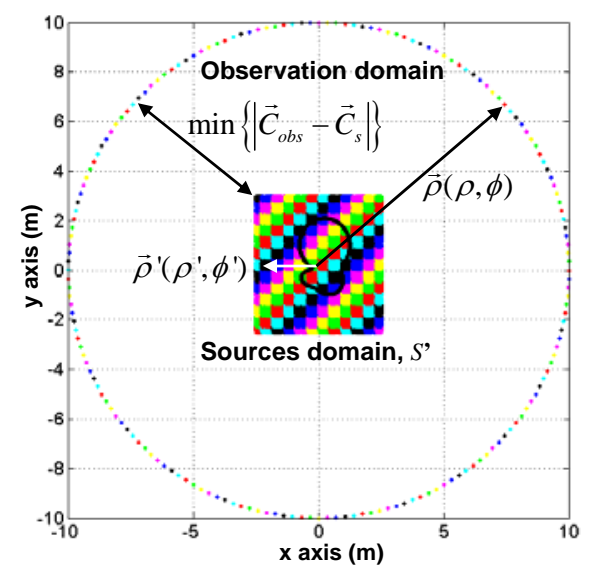

Fig. 1. Observation and sources domains, and FMM grouping.

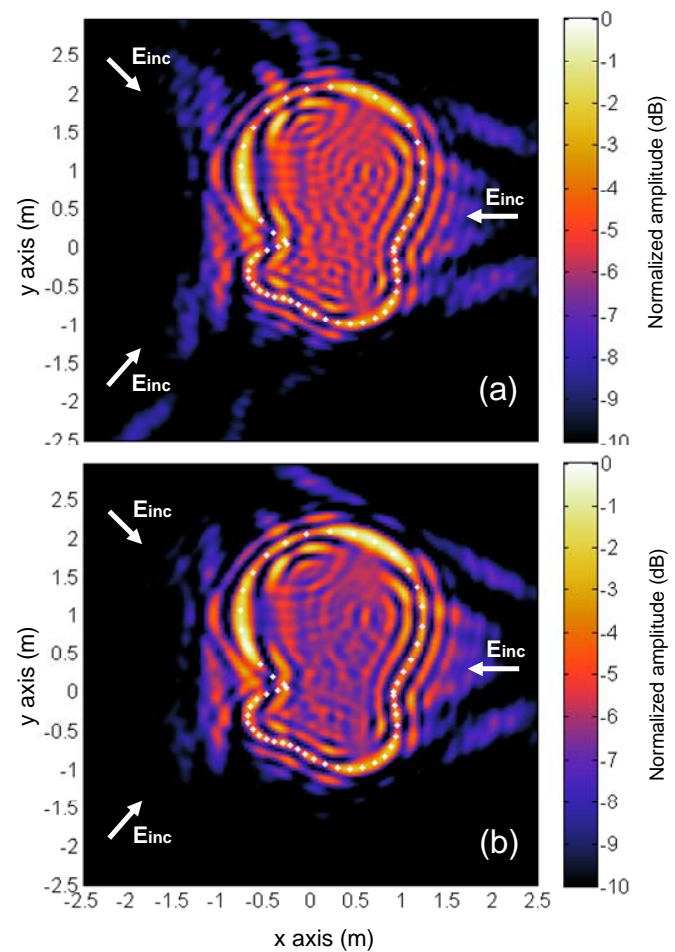

Fig. 2. Reconstructed equivalent currents (normalized amplitude, dB). (a) Using the Volumetric Source Reconstruction Method. (b) Using the Inverse FMM. 3 incidences and 16 frequencies are considered. The white dotted line represents the true profile.

Concerning the method accuracy, the retrieved equivalent electric currents using all the available information (16 frequencies and 3 incidences) are plotted in Fig. 2, generating a reasonable estimation of the object profile. It must be noted that the currents are combined coherently as indicated in (7).
In addition, when comparing the inverse FMM results Fig. 2 (a) to the ones given by the volumetric SRM Fig. 2 (b), no significant discrepancies are appreciated. The inverse FMM has also been tested for robustness against noise, plotting the reconstructed currents in Fig. 3: even for a $10 \mathrm{~dB}$ SNR, an acceptable profile estimation is achieved.

\begin{tabular}{l|c|c} 
& Volumetric SRM & Inverse FMM \\
\hline \hline 1 incidence, 1 freq. & $62 \mathrm{~s}$ & $1.5 \mathrm{~s}$ \\
1 inc., 16 freqs. & $1074 \mathrm{~s}$ & $21 \mathrm{~s}$ \\
3 inc., 16 freqs. & $2874 \mathrm{~s}$ & $62 \mathrm{~s}$ \\
& \multicolumn{2}{c}{ Table 1. Calculation time. }
\end{tabular}

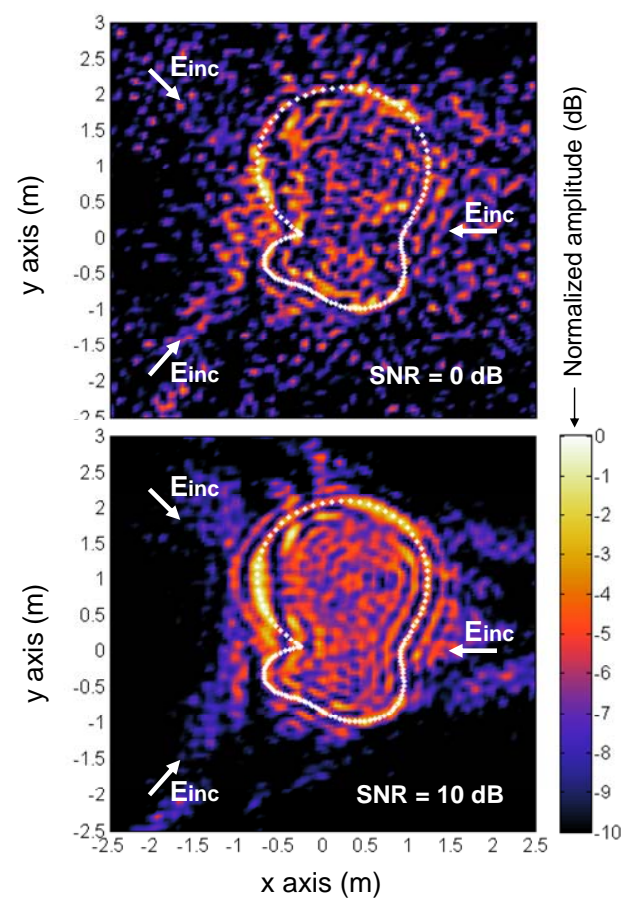

Fig. 3. Reconstructed equivalent currents (normalized amplitude, $\mathrm{dB}$ ) using 3 incidences and 16 frequencies for different SNR values. The white dotted line represents the true profile.

\section{B. Reconstruction of three metallic objects}

In a second example, the method's capability for recovering the metallic profile of several objects is tested. The objectsunder-test are the same as in [12], with an electric size of around $5 \lambda$ at the highest working frequency. 16 frequencies from 700 to $1000 \mathrm{MHz}$ are selected, resulting in a $35 \%$ bandwidth with respect to the center $850 \mathrm{MHz}$ frequency. The objects are illuminated by TM-polarized plane waves coming from 8 directions $\left(0^{\circ}\right.$ to $315^{\circ}$, in $45^{\circ}$-steps). The scattered field is evaluated in a circumference of $\rho=10 \mathrm{~m}$, sampled each $\Delta \phi=1^{\circ}$. It is assumed that the object-under-test profile is constant in the $\mathrm{z}$ direction, so again a 2D Method-of-Moments code has been used to evaluate the scattered fields. Noise is added with SNR of $40 \mathrm{~dB}$.

The equivalent currents are reconstructed from the calculated scattered field in a square domain of $6 \times 6 \mathrm{~m}$, discretized in $0.03 \times 0.03 \mathrm{~m}$ square patches, corresponding to $0.1 \times 0.1 \lambda$ at $1 \mathrm{GHz}$ (the highest frequency). This corresponds to an inverse problem with 360 equations (field samples) and 40401 unknowns (equivalent currents). If using all the 
available information (8 incidences and 16 frequencies), 128 inverse problems must be solved.

The inverse FMM technique is used to calculate the currents. As the number of observation points is low, no grouping is done on this domain. In the case of the reconstruction domain, the problem is divided in groups of 0.4 x $0.4 \mathrm{~m}$ (i.e. $1.33 \times 1.33 \lambda$ at $1 \mathrm{GHz}$ ), resulting in 225 source groups, a value not too far from the square root of the number of source points. The 128 inverse problems are solved in $\sim 5$ min, implying $2.3 \mathrm{~s}$ per problem.

The combined currents (7) for different frequencies and incidences [2] are plotted in Fig. 4. The presence of three objects is a more challenging profile reconstruction problem, requiring the combination of 8 incidences for an accurate profile reconstruction (Fig. 4 (b)). Nevertheless, even with 4 incidences, the profile can be inferred.

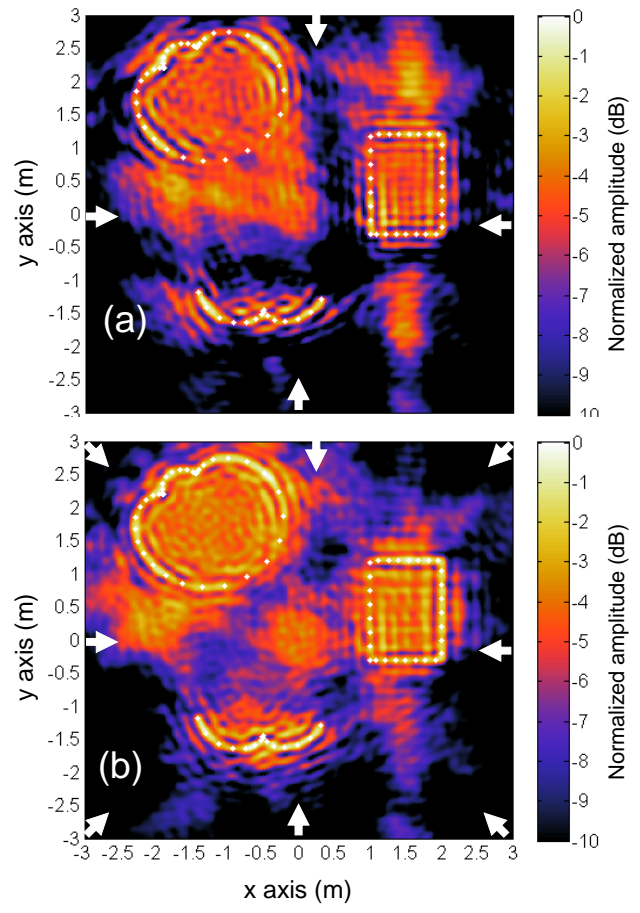

Fig. 4. Retrieved profile combining the equivalent currents for different incidences and frequencies. (a) 16 frequencies and 4 incidences. (b) 16 frequencies and 8 incidences. The white arrows indicate the arrival direction of the incident plane waves, and the white dotted line, the objects' profiles.

\section{CONCLUSION}

An inverse FMM for reducing the computational cost of inverse electromagnetic scattering problems has been presented. The main contribution is the possibility of solving an inverse problem in a forward fashion, without compromising the profile reconstruction accuracy, but also reducing the calculation time by 40 times. The proposed technique is of a special interest in these imaging systems which require fast processing of the information in order to provide an immediate diagnostics of the object-under-test.

\section{ACKNOWLEDGEMENTS}

The authors would like to acknowledge Ms. Sarah Rappaport for her suggestions and corrections regarding the readability of the manuscript.

\section{REFERENCES}

[1] E.C. Fear, X. Li, S.C. Hagness, M. A. Stuchly, "Confocal microwave imaging for breast cancer detection: localization of tumors in three dimensions," IEEE Transactions on Biomedical Engineering, Vol. 49, No. 8, pp 812-822. 2009.

[2] J.A. Martínez-Lorenzo, C. M. Rappaport, F. Quivira, "Physical Limitations on Detecting Tunnels Using Underground-Focusing Spotlight Synthetic Aperture Radar," IEEE Transactions on Geoscience and Remote Sensing, Vol. 49, No. 1, pp. 65-70, Jan. 2011.

[3] Reza Firoozabadi, Eric L. Miller, Carey M. Rappaport, Ann W. Morgenthaler, "Subsurface Sensing of Buried Objects Under a Randomly Rough Surface Using Scattered Electr. Field Data,” IEEE Trans. on Geoscience and Remote Sensing, Vol. 45, No. 1, pp. 104-117, Jan. 2007.

[4] D.M. Sheen, D.L. McMakin, T.E. Hall, "Three-dimensional millimeterwave imaging for concealed weapon detection," IEEE Transactions on Microwave Theory and Tech., Vol. 49, No. 9. pp. 1581-1592. 2001.

[5] M. Farmahini et al., "Fast and Accurate Cascaded Particle Swarm Optimization Method for solving 2-D Inverse Scattering Problems", Applied Computational Electromag. Soc. ACES. Vol. 24, No. 5, 2009.

[6] M. Çayören., I. Akduman, A. Yapar, L. Crocco, "A new algorithm for the shape reconstruction of perfectly conducting objects", Inverse Problems 23 (2007) - Ins. of Physics Publ, pp. 2087-1100. April 2007.

[7] Jose A. Martinez, Borja Gonzalez, Carey Rappaport, Javier G. Meana, Antonio G. Pino, "Reconstructing Distortions on Reflector Antennas With the Iterative-Field-Matrix Method Using Near-Field Observation Data," IEEE Trans. Ant. Prop., Vol. 59, No. 6, pp. 2434-2437, June 2011.

[8] C. Y. Lin, Y. W. Kiang, "Inverse scattering for conductors by the equivalent source method", IEEE Transactions on Antennas and Propagation, vol. 44, no. 3, pp. 310-316., March 1996.

[9] S. Caorsi, G. L. Gragnani, M. Pastorino, "Two-Dimensional Microwave Imaging by a Numerical Inverse Scattering Solution," IEEE Trans. on Microwave Theory and Tech., Vol. 38, No. 8, pp. 981-989, Aug. 1990.

[10] Matteo Pastorino, Andrea Massa, Salvatore Caorsi, "A Microwave Inverse Scattering Technique for Image Reconstruction Based on a Genetic Algorithm”, IEEE Transactions on Instrumentation and Measurement, Vol. 49, No. 3, pp. 573-578, June 2000.

[11]C. Estatico, G. Bozza, M. Pastorino, A. Randazzo, "An Inexact-Newton method for microwave reconstruction of strong scatterers," IEEE Antennas and Wireless Propagation Letters, vol. 5, pp. 61-64, 2006.

[12] Yuri Álvarez, Belén A. Casas, Cebrián García, Fernando Las-Heras, "Geometry Reconstruction of Metallic Bodies Using the Sources Reconstruction Method," IEEE Antennas and Wireless Propagation Letters. Vol. 9, pp. 1197-1200, 2009.

[13] Ronald Coifman, Vladimir Rokhlin, Stephen Wandzuraz, "The Fast Multipole Method for the Wave Equation: A Pedestrian Prescription," IEEE Antennas and Prop. Mag., Vol. 35, No. 3, pp. 7-11. June 1993.

[14]J. A. López-Fernández, M. L. Portugués, J. M. Taboada, H. J. Rice, F. Obelleiro, "HP-FASS: a hybrid parallel fast acoustic scattering solver," Intl. Journal of Comp. Maths, Vol. 88, Iss. 9, pp. 1960-1968, March 2011.

[15]W. C. Chew, T. J. Cui, J. M. Song, "A FAFFA-MLFMA Algorithm for Electromagnetic Scattering," IEEE Transactions on Antennas and Propagation, Vol. 50, No. 11, pp. 1640-1649, November 2002.

[16] P. Wang, M. Xia, L. Zhou, "Analysis of scattering by combined conducting and dielectric bodies using single integral equation method and fast multipole method," Proc. Microwave Conference, 2005. APMC 2005. Asia-Pacific Conference. Suzhou, China, 4-7 Sept. 2005. pp. 1-4.

[17]D. Vandde Ginste, D. De Zutter, L. Knkckaert, "New upper bounds to determine the sampling rate in 2-D MLMFA," 2009 IEEE Antennas and Prop. Society Intl. Symp., Charleston, USA, June 1-5, 2009. pp. 1-4.

[18]A. Boag, "A Fast Multilevel Domain Decomposition Algorithm for Radar Imaging," IEEE Transactions on Antennas and Propagation, Vol. 49, No. 4, pp. 666-671, April 2001.

[19]T. F. Eibert, C. H. Schmidt, "Multilevel Fast Multipole Accelerated Inverse Equivalent Current Method Employing Rao-Wilton-Glisson Discretization of Electric and Magnetic Surface Currents," IEEE Trans. on Antennas and Propagation, Vol. 57, No. 4, pp. 1178-1185, April 2009.

[20] Y. Alvarez, F. Las-Heras, M. R. Pino, Jesús A. López, "Acceleration of the Sources Reconstruction Method via the Fast Multipole Method,” 2008 IEEE International Symposium on Antennas and Propagation (APS'08). San Diego, CA (USA). 5-12 July 2008. 